\title{
Mesoscopic Mechanism of Adiabatic Charge Transport
}

\author{
F. Zhou, ${ }^{1}$ B. Spivak, ${ }^{2}$ and B. Altshuler ${ }^{1,3}$ \\ ${ }^{1}$ Physics Department, Princeton University, Princeton, New Jersey 08544 \\ ${ }^{2}$ Physics Department, University of Washington, Seattle, Washington 98195 \\ ${ }^{3}$ NEC Research Institute, 4 Independence Way, Princeton, New Jersey 08540
}

(Received 17 September 1998)

\begin{abstract}
We consider adiabatic charge transport through mesoscopic metallic samples caused by a periodically changing external potential. We find that both the amplitude and the sign of the charge transferred through a sample per period are random sample specific quantities. The characteristic magnitude of the charge is determined by the quantum interference. [S0031-9007(98)08205-2]
\end{abstract}

PACS numbers: 73.23.-b, 72.15.Rn

Let us apply an external potential $\phi(\mathbf{r}, t)$, which is changing slowly and periodically in time to a metallic sample. This potential causes finite net charge $Q$ transported across the sample per period. This phenomenon, known as adiabatic charge transport [1], has been investigated in several papers [1-4] for a closed system characterized by its ground state wave function corresponding to the instantaneous value of the external potential $\phi(\mathbf{r}, t)$. However, in real experimental situations exact eigenfunctions of electrons are ill defined: The electron energy levels are broadened due to inelastic processes at $T \neq 0$, and, in the case of an open system, are further broadened due to finite dwell time.

Here we present a theory of adiabatic charge transport in "open mesoscopic systems." We demonstrate that at low $T$ both the magnitude and the sign of $Q$ are sample specific quantities. The typical value of $Q$ in disordered (chaotic) systems turns out to be determined by quantum interference effects. We evaluate this value and find that it is much larger than the one in ballistic systems. This enhancement manifests of the well-known fact that at low temperatures, all electronic characteristics of mesoscopic samples are extremely sensitive to changes in the scattering potential [5-8].

Let us start with a qualitative picture of the mesoscopic adiabatic charge transport. The wave functions of electrons in disordered systems exhibit sample specific spatial fluctuations. Therefore, the spatial electron density profile is changing slowly in time, together with the external potential $\phi(\mathbf{r}, t)$. According to the continuity relation, variation of the charge density in time requires currents in the system. The question arises: What is the condition for a total charge transfer during one period to be nonzero? Let the pumping potential $\phi(\mathbf{r}, t)$ be characterized by a finite set of functions $\mathbf{g}(t)=\left\{g_{\alpha}(t)\right\}, \alpha=1, \ldots, m$, which are periodic with the same period $t_{0}$ :

$$
\phi(\mathbf{r}, t)=\phi(\mathbf{r}, \mathbf{g}(t))=\sum_{\alpha} \phi_{\alpha}(\mathbf{r}) g_{\alpha}(t)
$$

The time evolution of the functions $\mathbf{g}(t)$ represents a motion of a point in $m$ dimensional space $\mathcal{M}$. Because of the periodicity of $\phi(\mathbf{r}, t)$, the trajectory $C$ of this point is closed. The above-mentioned currents lead to $Q \neq 0$, provided $C$ encloses a finite area in $\mathcal{M}$. This requires that $m \geq 2$.

To calculate $Q$ we will use the Keldysh technique for the Green function matrix equation [9]

$$
\begin{gathered}
\left(i \partial_{t}-H_{0}-\phi(\mathbf{r}, t)\right) \hat{G}\left(\mathbf{r}, \mathbf{r}^{\prime} ; t, t^{\prime}\right)-I_{e-\mathrm{ph}}(\{\hat{G}\}) \\
=\delta\left(\mathbf{r}-\mathbf{r}^{\prime}\right) \delta\left(t-t^{\prime}\right),
\end{gathered}
$$

where $\hat{G}$ is a $2 \times 2$ matrix, $G_{11}=0, G_{12}=G^{A}, G_{21}=$ $G^{R}$, and $G_{22}=G^{K}$, with $G^{R, A, K}$ being the retarded, advanced, and Keldysh Green functions, respectively. $H_{0}$ is the Hamiltonian for electrons which includes impurity scattering potentials, and $I_{e-\mathrm{ph}}$ denotes the electronphonon collision integral. The solution of Eq. (2) can be expanded in terms of the changing rate of the external field $\dot{\phi}(\mathbf{r}, t)$, provided the time of the electron diffuses across the sample is shorter than the period of the external potential $t_{0}$. In general, $\hat{G}\left(\mathbf{r}, \mathbf{r}^{\prime} ; t, t\right)$ depends on the value of the potential $\phi\left(\mathbf{r}, t^{\prime}\right)$ at all the previous times $t^{\prime} \leq t$. However, in the first order adiabatic approximation $G\left(\mathbf{r}, \mathbf{r}^{\prime} ; t, t\right)$ is determined only by the external potential and its first time derivative at time $t$, i.e., $\hat{G}\left(\mathbf{r}, \mathbf{r}^{\prime} ; t, t\right)=$ $\hat{G}(\{\phi(\mathbf{r}, t)\},(\dot{\phi}(\mathbf{r}, t)\})$. The local time dependence in this approximation allows us to express the charge transfer $Q_{i}$ per period $t_{0}$ along the $i$ th direction as

$$
Q_{i}=\frac{e}{L_{i} m} \int_{0}^{t_{0}} d t \operatorname{Tr}\left\{P_{i} G^{K}(t, t)\right\}=e \int_{C} \omega_{\alpha}^{i}(\mathbf{g}) d g_{\alpha},
$$

where $P_{i}$ is the $i$ th component of the momentum operator, $L_{i}$ is the dimension of the sample along the $i$ th direction, $i=x, y, z$, Tr means integration over the space coordinates, and

$$
\omega_{\alpha}^{i}(\mathbf{g})=\frac{\partial}{\partial \dot{g}_{\alpha}} \operatorname{Tr}\left\{\frac{P_{i}}{m L_{i}} G^{K}(t, t)\right\} .
$$

Equation (4) is valid only in the leading order in $\Omega=$ $2 \pi / t_{0}$. We have introduced differential $1-$ form $\omega_{\alpha}^{i}(\mathbf{g})$ on the $m$ dimensional space $\mathcal{M}$. Using Stokes theorem one can convert 1 - form integrals along a trajectory $C$ into $2-$ form integrals over any surface $S$ 
spanning $C$,

$$
\begin{aligned}
Q_{i} & =e \int_{S} d g_{\alpha} \wedge d g_{\beta} \pi_{\alpha \beta}^{i}(\mathbf{g}), \\
\pi_{\alpha \beta}^{i}(\mathbf{g}) & =\left\{\frac{\partial}{\partial g_{\alpha}} \frac{\partial}{\partial \dot{g}_{\beta}}-\frac{\partial}{\partial g_{\beta}} \frac{\partial}{\partial \dot{g}_{\alpha}}\right\} \operatorname{Tr}\left\{\frac{P_{i}}{2 m L_{i}} G^{K}(t, t)\right\} .
\end{aligned}
$$

In an isolated quantum mechanical system, the 2-form $\pi_{\alpha \beta}^{i}$ corresponds to the generalized adiabatic curvature of discrete eigenstates discussed in [3]. The wedge product is skew symmetric, i.e., $d g_{\alpha} \wedge d g_{\beta}=-d g_{\beta} \wedge d g_{\alpha}$.

In the zero order adiabatic approximation Keldysh Geen function $G_{\epsilon}^{K}\left(\mathbf{r}, \mathbf{r}^{\prime}, t\right)$ can still be expressed through retarded and advanced ones $G_{\epsilon}^{R, A}\left(\mathbf{r}, \mathbf{r}^{\prime}, t\right)$ and Fermi distribution function $n_{F}(\epsilon)$

$$
G_{\epsilon}^{K}=\left(G_{\epsilon}^{R}-G_{\epsilon}^{A}\right)\left[1-2 n_{F}(\epsilon)\right],
$$

where matrix elements of $\hat{G}_{\epsilon}$ correspond to the instantaneous Hamiltonian $H_{0}+\phi(\mathbf{r}, \mathbf{g})$,

$$
\hat{G}_{\epsilon}\left(\mathbf{r}, \mathbf{r}^{\prime}, t\right)=\int d t^{\prime} \hat{G}\left(\mathbf{r}, \mathbf{r}^{\prime} ; t-\frac{t^{\prime}}{2}, t+\frac{t^{\prime}}{2}\right) e^{i \epsilon t^{\prime}}
$$

and depend on time only through $\mathbf{g}(t)$.

According to Eqs. (2), (7), the first order correction to the adiabatic approximation for $G^{K}$ is

$$
\begin{aligned}
\delta G_{\epsilon}^{K}\left(\mathbf{r}, \mathbf{r}^{\prime}, t\right)= & i \int d \mathbf{r}^{\prime \prime}\left\{\left[\frac{1}{2}-n_{F}(\epsilon)\right]\left(\Gamma_{R R}-\Gamma_{A A}\right)\right. \\
& \left.-\frac{\partial n_{F}(\epsilon)}{\partial \epsilon} \Gamma_{R A}\right\}
\end{aligned}
$$

where for any $p=(R, A), q=(R, A)$,

$$
\begin{aligned}
\Gamma_{p q}\left(\mathbf{r}, \mathbf{r}^{\prime}, \mathbf{r}^{\prime \prime},(t)=\right. & 2 G_{\epsilon}^{p}\left(\mathbf{r}, \mathbf{r}^{\prime \prime}, t\right) \partial_{t} G_{\epsilon}^{p}\left(\mathbf{r}^{\prime \prime}, \mathbf{r}^{\prime}, t\right) \delta_{p q} \\
& +G_{\epsilon}^{p}\left(\mathbf{r}, \mathbf{r}^{\prime \prime}, t\right) \partial_{t} \phi\left(\mathbf{r}^{\prime \prime}, t\right) G_{\epsilon}^{q}\left(\mathbf{r}^{\prime \prime}, \mathbf{r}^{\prime}, t\right) .
\end{aligned}
$$

The contribution of the first two terms in Eq. (8) to $Q_{i}$ can be neglected provided $L_{i}$ is much bigger than the elastic mean free path $l$. Using Eq. (8) we present $\pi_{\alpha \beta}^{i}(\mathbf{g})$, Eq. (5) as

$$
\begin{aligned}
\pi_{\alpha \beta}^{i}(\mathbf{g}) & =\frac{\partial \Xi_{i}(\beta, \mathbf{g})}{\partial g_{\alpha}(t)}-\frac{\partial \Xi_{i}(\alpha, \mathbf{g})}{\partial g_{\beta}(t)} \\
\Xi_{i}(\alpha, \mathbf{g}) & =\left.\int d \mathbf{r} d \mathbf{r}_{1} \phi_{\alpha}\left(\mathbf{r}_{1}\right) \frac{1}{2 m L_{i}}\left(\frac{\partial}{\partial r_{i}}-\frac{\partial}{\partial r_{i}^{\prime}}\right) \int d \epsilon \frac{\partial n_{F}(\boldsymbol{\epsilon})}{\partial \epsilon} G_{\epsilon}^{R}\left(\mathbf{r}, \mathbf{r}_{1}, t\right) G_{\epsilon}^{A}\left(\mathbf{r}_{1}, \mathbf{r}^{\prime}, t\right)\right|_{\mathbf{r} \rightarrow \mathbf{r}^{\prime}}
\end{aligned}
$$

Because of the disorder, the charge $Q_{i}$ is a random sample-specific quantity. To characterize $Q_{i}$, we calculate its average $\left\langle Q_{i}\right\rangle$ and variance $\left\langle\left(\delta Q_{i}\right)^{2}\right\rangle(\langle\rangle$ stands for the averaging over realizations of the random potential). In the following, we assume that $L_{\phi} \gg L_{z}, L_{x}, L_{y} \gg l$, where $L_{\phi}$ is the electron dephasing length. In this case one can express $\langle Q\rangle$ and $\left\langle\left(\delta Q_{i}\right)^{2}\right\rangle$ through $\left\langle\pi_{\alpha \beta}^{i}(\mathbf{g})\right\rangle$ and variance $\prod_{\alpha \beta \alpha^{\prime} \beta^{\prime}}^{i}\left(\mathbf{g}, \mathbf{g}^{\prime}\right)=\left\langle\delta \pi_{\alpha \beta}^{i}(\mathbf{g}) \delta \pi_{\alpha^{\prime} \beta^{\prime}}^{i}\left(\mathbf{g}^{\prime}\right)\right\rangle$. These quantities can be evaluated in a standard way [10]. Following Eq. (10), $\left\langle\pi_{\alpha \beta}^{i}\right\rangle$ can be calculated in terms of diagrams in Fig. 1a,

$$
\left\langle\pi_{\alpha \beta}^{i}(\mathbf{g})\right\rangle=\frac{\nu D_{0}}{2 \mu_{F} L_{i}} \int d \mathbf{r} d \mathbf{r}^{\prime} \phi_{\alpha}(\mathbf{r}) \phi_{\beta}\left(\mathbf{r}^{\prime}\right) \frac{\partial \mathcal{D}\left(\mathbf{r}, \mathbf{r}^{\prime}\right)}{\partial r_{i}},
$$

where $\nu$ is the mean density of states in the metal, $\mu_{F}$ is the Fermi energy, and $D_{0}$ is the electron diffusion coefficient. Note that $\left\langle\pi_{\alpha \beta}^{i}\right\rangle$ does not depend on the external field, i.e., on $\mathbf{g}$ in the leading order of $\phi / \mu_{F}$.

$\Pi_{\alpha \beta \alpha^{\prime} \beta^{\prime}}^{i}$ (diagrams Fig. 1b) can be presented in the form

$$
\begin{aligned}
\Pi_{\alpha \beta \alpha^{\prime} \beta^{\prime}}^{i}= & \tilde{\Pi}_{\alpha \beta \alpha^{\prime} \beta^{\prime}}^{i}+\tilde{\Pi}_{\beta \alpha \beta^{\prime} \alpha^{\prime}}^{i}-\tilde{\Pi}_{\alpha \beta \beta^{\prime} \alpha^{\prime}}^{i}-\tilde{\Pi}_{\beta \alpha \alpha^{\prime} \beta^{\prime}}^{i} \\
\tilde{\Pi}_{\alpha \beta \alpha^{\prime} \beta^{\prime}}^{i}\left(\mathbf{g}, \mathbf{g}^{\prime}\right)= & \frac{D_{0}^{2}}{4 L_{i}^{2}} \int d \epsilon d \omega \frac{\partial n_{F}(\epsilon+\omega / 2)}{\partial \epsilon} \frac{\partial n_{F}(\epsilon-\omega / 2)}{\partial \epsilon} \\
& \times \int d \mathbf{r}_{1} d \mathbf{r}_{1}^{\prime} d \mathbf{r} d \mathbf{r}^{\prime} \phi_{\alpha}\left(\mathbf{r}_{1}\right) \phi_{\alpha^{\prime}}\left(\mathbf{r}_{1}^{\prime}\right) \nabla_{\mathbf{r}_{1}} \mathcal{D}\left(\mathbf{r}_{1}, \mathbf{r}\right) \frac{\partial^{2} \Lambda}{\partial g_{\beta} \partial g_{\beta^{\prime}}},
\end{aligned}
$$

which explicitly demonstrates antisymmetry of the pumping perturbations. $\Lambda$ is given as

$$
\begin{aligned}
\Lambda= & \operatorname{Re}\left[\mathcal{D}\left(\omega, \mathbf{r}, \mathbf{r}^{\prime} ; \mathbf{g}, \mathbf{g}^{\prime}\right) \mathcal{D}\left(\omega, \mathbf{r}^{\prime}, \mathbf{r} ; \mathbf{g}, \mathbf{g}^{\prime}\right)\right] \nabla_{\mathbf{r}_{1}^{\prime}} \mathcal{D}\left(\mathbf{r}_{1}^{\prime}, \mathbf{r}^{\prime}\right)+2 \mathcal{D}\left(\omega, \mathbf{r}, \mathbf{r}^{\prime} ; \mathbf{g}, \mathbf{g}^{\prime}\right) \mathcal{D}^{*}\left(\omega, \mathbf{r}^{\prime}, \mathbf{r} ; \mathbf{g}, \mathbf{g}^{\prime}\right) \nabla_{\mathbf{r}_{1}^{\prime}} \mathcal{D}\left(\mathbf{r}_{1}^{\prime}, \mathbf{r}^{\prime}\right) \\
& +2 \mathcal{D}\left(\omega, \mathbf{r}, \mathbf{r}^{\prime} ; \mathbf{g}, \mathbf{g}^{\prime}\right) \mathcal{D}^{*}\left(\omega, \mathbf{r}^{\prime}, \mathbf{r} ; \mathbf{g}, \mathbf{g}^{\prime}\right) \nabla_{\mathbf{r}_{1}^{\prime}} \mathcal{D}\left(\mathbf{r}_{1}^{\prime}, \mathbf{r}\right) .
\end{aligned}
$$

$\mathcal{D}\left(\mathbf{r}, \mathbf{r}^{\prime}\right)$ and $\mathcal{D}\left(\omega, \mathbf{r}, \mathbf{r}^{\prime} ; \mathbf{g}, \mathbf{g}^{\prime}\right)$ satisfy the following equations:

$$
\begin{gathered}
D_{0} \nabla^{2} \mathcal{D}\left(\mathbf{r}, \mathbf{r}^{\prime}\right)=\delta\left(\mathbf{r}-\mathbf{r}^{\prime}\right), \\
\left\{i \omega+i \delta \phi+D_{0} \nabla^{2}\right\} \mathcal{D}\left(\omega, \mathbf{r}, \mathbf{r}^{\prime} ; \mathbf{g}, \mathbf{g}^{\prime}\right)=\delta\left(\mathbf{r}-\mathbf{r}^{\prime}\right),
\end{gathered}
$$




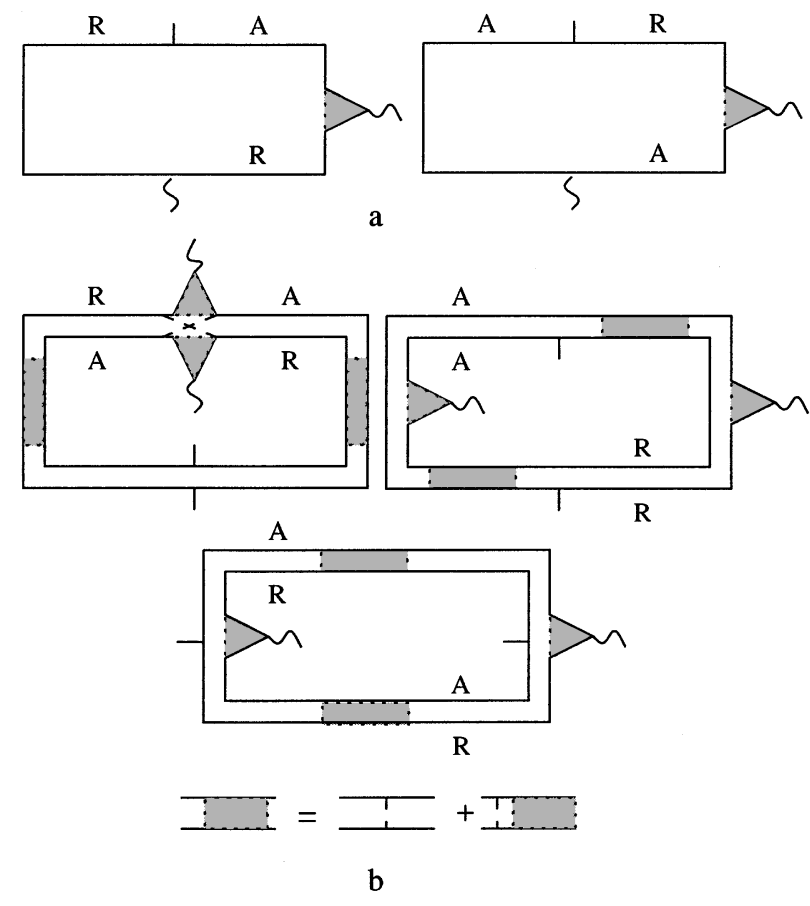

FIG. 1. (a) Diagrams for $\left\langle\pi_{\alpha \beta}^{i}(\mathbf{g})\right\rangle$; (b) diagrams for $\Pi_{\alpha \beta \alpha^{\prime} \beta^{\prime}}^{i}\left(\mathbf{g}, \mathbf{g}^{\prime}\right)$. Solid lines represent retarded $(R)$ and advanced $(A)$ electron Green functions, dashed lines denote the impurity averaging, and wavy lines represent the external potential. Shaded triangles stand for $\mathcal{D}\left(\mathbf{r}, \mathbf{r}^{\prime}\right)$, shaded boxes for diffusions $\mathcal{D}\left(\omega, \mathbf{r}, \mathbf{r}^{\prime} ; \mathbf{g}, \mathbf{g}^{\prime}\right)$. In (b), the shaded triangles with two dashed lines represent Hikami boxes.

where $\delta \phi\left(\mathbf{r}, \mathbf{g}, \mathbf{g}^{\prime}\right)=\phi\left(\mathbf{r}, \mathbf{g}^{\prime}\right)-\phi\left(\mathbf{r}, \mathbf{g}^{\prime}\right)$. For Eqs. (14) and (15), we use usual boundary conditions: $\mathcal{D}\left(\mathbf{r}, \mathbf{r}^{\prime}\right)=$ $\mathcal{D}\left(\epsilon-\epsilon^{\prime}, \mathbf{r}, \mathbf{r}^{\prime}\right)=0$ when $\mathbf{r}$ or $\mathbf{r}^{\prime}$ is at open boundaries $[11,12]$.

Let us now consider the sample sketched in Fig. 2 with two gates (labeled by $\alpha=1,2$ ), biased with ac voltages of the same frequency and with a phase shift $\delta=\delta_{1}-\delta_{2}$,

$$
V_{\alpha}(t)=V_{0} \sin \left(\Omega t+\delta_{\alpha}\right) .
$$

In this case, $m=2$. Let us assume that the potential induced in the metal by the voltages $V_{\alpha}$ is screened with a screening length $r_{0}$ much less than $L_{x}$ and

$$
\begin{aligned}
g_{\alpha}(t) & =\sin \left(\Omega t+\delta_{\alpha}\right), \\
\phi_{\alpha}(\mathbf{r}) & =\frac{C V_{0} r_{0}}{L_{y} W} \theta\left(r_{0}-x\right) \theta\left(\frac{W^{2}}{4}-\left(Z_{\alpha}-z\right)^{2}\right),
\end{aligned}
$$

where $C$ is the capacitance of the gate, $W \gg r_{0}$ is the width of the gate along the $z$ direction, $Z_{1,2}$ are the $z$ coordinates of the center of the gate 1,2 ; and $\theta(z)$ is the step function: $\theta(z \geq 0)=1$ while $\theta(z<0)=0$.

To evaluate $\left\langle Q_{z}\right\rangle$ in the leading order in $V_{0} / \mu_{F} \ll 1$, one has to substitute Eqs. (11) and (17) into Eqs. (5) and take into account that

$$
\int_{S} d g_{\alpha} \wedge d g_{\beta}=\int_{0}^{t_{0}} d t\left\{\partial_{t} g_{\alpha}(t) g_{\beta}(t)-\partial_{t} g_{\beta}(t) g_{\alpha}(t)\right\} \text {. }
$$

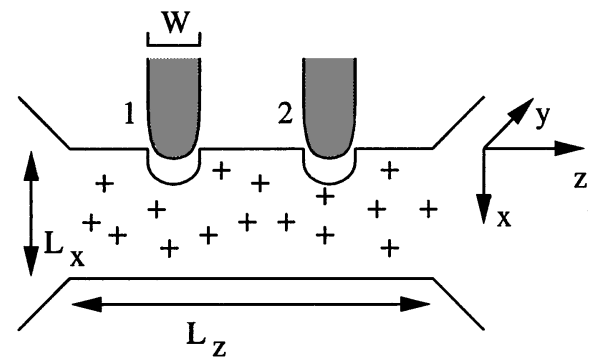

FIG. 2. Geometry of the sample. Shaded bars represent gates 1 and 2; crosses represent random scatters.

Given the volume of the sample $v=L_{x} L_{y} L_{z}$ and the total number of electrons inside the sample $N$, we present $\left\langle Q_{z}\right\rangle$ as

$$
\left\langle Q_{z}\right\rangle=f_{0} e \sin (\delta)\left(\frac{C V_{0} r_{0}^{2}}{v \mu_{F}}\right)^{2} N .
$$

$f_{0} \sim 1$ is a geometry-dependent factor.

To determine $\Pi_{\alpha \beta \alpha^{\prime} \beta^{\prime}}^{i}$ one has to solve Eq. (15). When

$$
\frac{C V_{0} r_{0}^{2}}{v} \ll E_{T}=\frac{D_{0}}{L_{z}^{2}},
$$

this can be done by using perturbation theory with respect to $g_{\alpha}(t)$. Keeping only the bilinear in $\mathbf{g}$ and $\mathbf{g}^{\prime}$ contributions to $\mathcal{D}\left(\omega, \mathbf{r}, \mathbf{r}^{\prime} ; \mathbf{g}, \mathbf{g}^{\prime}\right)$, we express the solution of Eq. (15) in terms of $\mathcal{D}^{0}\left(\omega, z, z^{\prime}\right)$, the $\mathbf{g}$ independent solution of Eq. (15) with $\phi(\mathbf{r}, \mathbf{g})=0$. As usual, in the quasi-one-dimensional case, we neglect $x, y$ dependences of $\mathcal{D}^{0}$. Using Eq. (12), we express $\Pi_{\alpha \beta \alpha^{\prime} \beta^{\prime}}^{z}\left(\mathbf{g}, \mathbf{g}^{\prime}\right)$ in terms of $\mathcal{D}^{0}$. It is important that $\Pi_{\alpha \beta \alpha^{\prime} \beta^{\prime}}^{z}$ is independent of $\mathbf{g}, \mathbf{g}^{\prime}$ in the leading order of $C V_{0} r_{0}^{2} / v E_{T} \ll 1$. Thus, according to Eq. (5), $\sqrt{\left\langle\left(\delta Q_{z}\right)^{2}\right\rangle}$ is proportional to the area $S$ enclosed by the trajectory $C$. As a result for $C V_{0} r_{0}^{2} / v \ll E_{T}$,

$$
\left\langle\left(\delta Q_{z}\right)^{2}\right\rangle=(e \sin \delta)^{2}\left(\frac{C V_{0} r_{0}^{2}}{v E_{T}}\right)^{4} f_{1}\left(\frac{T}{E_{T}}\right) .
$$

Here the function $f_{1}(\eta)$ has the following asymptotics:

$$
f_{1}(\eta) \propto \begin{cases}1, & \eta \ll 1, \\ \eta^{-1}, & \eta \gg 1 .\end{cases}
$$

In the limit $C V_{0} r_{0}^{2} / v \gg E_{T}, \pi_{\alpha \beta}^{z}(\mathbf{g})$ is a random quantity in 2D space $\mathcal{M}$ of $\{\mathbf{g}\}$ with the "correlation length" $\left|\delta \mathbf{g}_{c}\right| \sim E_{T} \boldsymbol{v} / C V_{0} r_{0}^{2}$ (which is much less than unity). $\sqrt{\left\langle\left(\delta Q_{z}\right)^{2}\right\rangle}$ is determined by the amount of "flux" of random $\pi_{\alpha \beta}^{z}(\mathbf{g})$ field that threads the loop $C$ and should increase slower than the enclosed area $S$ itself. This limit will be considered elsewhere.

It follows from Eqs. (19) and (21) that the standard deviation of $Q_{z}$ far exceeds its average, provided $N \gg G_{z}^{2}$ ( $G_{z}$ is the dimensionless conductance in the $z$ direction), and the amplitude of the external perturbation is not too large. In this case the value of $Q_{z}$ is entirely determined by quantum interference effects. In the opposite limit, $Q_{z}$ can be characterized by its average given by Eq. (19).

According to Eq. (19), $\left\langle Q_{z}\right\rangle$ is proportional to $\sin \delta$, i.e., this quantity changes sign together with $\delta$ and vanishes at 
$\delta=0$. In fact, that is correct for the charge transfer $Q_{z}$ in a specific sample of given realization of disorder. One can see this from Eq. (5), taking into account that $\pi_{\alpha \beta}^{i}(\mathbf{g})$ is a random quantity independent of $\mathbf{g}$ when $\mathbf{g}$ is small. More generally, the charge transfer $Q$ changes sign when $\delta \rightarrow$ $-\delta$ for arbitrary amplitude of the external perturbation, although the simple $\delta$ dependence in Eqs. (19) and (21) is valid only in the weak external field. Indeed, $T$ invariance requires that changing the direction of the trajectory $C$ in the space $\mathcal{M}$ (which corresponds to changing sign of $\delta$ for the case of two gates) should result in the change of sign of the charge: $Q_{\hookleftarrow}=-Q_{\hookrightarrow}$ where $\hookleftarrow(\hookrightarrow)$ corresponds to clockwise (counterclockwise) motion along the same closed trajectory $C$. In the presence of magnetic field, this identity acquires a form

$$
Q_{\hookleftarrow}(\mathbf{H})=-Q_{\hookrightarrow}(-\mathbf{H}) .
$$

Here $Q_{\hookleftarrow}(\mathbf{H})$ and $Q_{\hookrightarrow}(\mathbf{H})$ are charges which correspond to clockwise and counterclockwise motion along the same closed trajectory $C$ in the space $\mathcal{M}$ and $\mathbf{H}$ is the external magnetic field. When the external potential is weak, one can neglect the $\mathbf{g}$ dependence of $\pi_{\alpha \beta}^{i}$. In this limit, $Q$ is proportional to the area $S$ enclosed by trajectory $C$ and $\pi_{\alpha \beta}^{i}(\mathbf{g} \approx 0)$. Thus, Eq. (23) indicates that in the vicinity of $\mathbf{g}=0 \pi_{\beta}^{i}(\mathbf{g})$ is an even function of $\mathbf{H}$. At small amplitudes of the external potential, this leads to $Q_{\hookleftarrow(\hookrightarrow)}(\mathbf{H})=Q_{\hookleftarrow(\hookrightarrow)}(-\mathbf{H})$, provided without magnetic fields the system is $T$ invariant.

As is usual in mesoscopic physics, the magnetic field dependence of $Q_{z}(H)$ exhibits random sample specific fluctuations with a characteristic period $\Delta H \sim \phi_{0} / L_{z} L_{x}$. Here $\Phi_{0}$ is the flux quanta (the magnetic field is applied along the $y$ direction). At high temperatures, when the dephasing length is shorter than the sample size, mesoscopic effects become exponentially small and $Q_{z}$ is determined by Eq. (19).

A different mesoscopic mechanism of the adiabatic charge transport has been discussed in [13]. Inelastic electron-phonon processes shift centers of mass of wave functions and thus contribute to $Q_{i}$. However, for open samples, this contribution is small compared with Eq. (21) as $\left(\tau_{e-\mathrm{ph}} E_{T}\right)^{-1} \ll 1$, where $\tau_{e \text {-ph }}$ is the electron-phonon inelastic scattering time.

In conclusion, we demonstrated that it is possible to speak about adiabatic quantum pumping in statistical systems. Note that the dc current discussed above is proportional to the frequency of the oscillations of the external potential $\Omega$. This distinguishes the considered above effect from the usual photovoltaic effect. The latter effect in the low frequency limit is dominated by the relaxations of nonequilibrium distribution of electrons in the presence of external field via electron-phonon inelastic processes [13]. Such a mechanism leads to a randomly directed dc current proportional to the absorption rate of the external field even in the presence of a single pumping gate voltage. It means that the photovoltaic dc current is proportional to
$\Omega^{2}$, as the frequency dependence of the absorption rate of the external field is. In an open sample in the low frequency limit, the photovoltaic current is smaller than the adiabatic current by a factor $\Omega / E_{T} \ll 1$.

We want to emphasize that the value of $Q$ in a finite open mesoscopic system is not quantized. An approximate quantization of $Q$ can be achieved in a Coulomb blockade regime, for a pumping of the charge through a quantum dot, which is weakly connected with the source and drain [1416]. $Q$ turns out to be rather well quantized provided the dimensionless conductance of the device is small. However, under these conditions, pumping is an entirely classical effect and has nothing to do with quantum interference.

We acknowledge useful discussions with I. Aleiner, C. Marcus, and T. Shutenko. Work of B. Spivak is supported by DMR, U.S. NSF under Grant No. DMR9205114. Work of F. Zhou is supported by ARO under Contract No. DAAG 55-98-1-0270.

Note added.-While preparing the manuscript we have learned about the paper of P. Brower where similar results were obtained.

[1] D. Thouless, Phys. Rev. B 27, 6083 (1983).

[2] M. V. Berry, Proc. R. Soc. London A 392, 45 (1984).

[3] J. E. Avron, A. Raveh, and B. Zur, Rev. Mod. Phys. 60, 873 (1988).

[4] Geometric Phases in Physics, in Advanced Series in Mathematical Physics (World Scientific, Singapore, 1989), edited by A. A. Shapere and F. Wilczek.

[5] B. Altshuler and B. Spivak, Pis'ma Zh. Eksp. Teor. Fiz. 42, 363 (1985) [JETP Lett. 42, 447 (1985)].

[6] S. Feng, P. A. Lee, and A. D. Stone, Phys. Rev. Lett. 56, 1560 (1986).

[7] B. Spivak and Z. Zyuzin, Mesoscopic Fluctuations of Current Density in Disordered Conductors, in Mesoscopic Phenomena in Solids (Elsevier Science Publishers, B.V., Amsterdam, 1991), edited by B. L. Altshuler, P. A. Lee, and R. A. Webb.

[8] B. Simons and B. Altshuler, Phys. Rev. Lett. 70, 4063 (1993); B. Simons and B. Altshuler, Phys. Rev. B 48, 5422 (1993).

[9] L. Keldysh, Zh. Eksp. Teor. Fiz. 47, 1515 (1964) [Sov. Phys. JETP 20, 1018 (1965)].

[10] A. A. Abrikosov, L. P. Gorkov, and I.E. Dzyaloshinski, Methods of Quantum Field Theory in Satistical Physics (Dover, New York, 1961).

[11] B. Altshuler, Pis'ma Zh. Eksp. Teor. Fiz. 41, 530 (1985) [JETP Lett. 41, 648 (1985)].

[12] P. A. Lee and D. Stone, Phys. Rev. Lett. 55, 1622 (1985).

[13] B. Spivak, F. Zhou, and M. T. Beal Monod, Phys. Rev. B 51, 13226 (1995).

[14] L.P. Kouwenhoven, A. T. Johnson, N. C. van der Varrt, C. J. P. M. Harmans, and C. T. Foxon, Phys. Rev. Lett. 67, 1626 (1991); Z. Phys. B 85, 381 (1991).

[15] H. Pothier, P. Lafarge, C. Urbina, D. Esteve, and M. H. Devoret, Europhys. Lett. 17, 249 (1992).

[16] I. L. Aleiner and A. V. Andreev, Phys. Rev. Lett. 81, 1286 (1998). 